\title{
Two Asian egg parasitoids of Halyomorpha halys (Stål) (Hemiptera, Pentatomidae) emerge in northern Italy: Trissolcus mitsukurii (Ashmead) and Trissolcus japonicus (Ashmead) (Hymenoptera, Scelionidae)
}

\author{
Giuseppino Sabbatini Peverieri', Elijah Talamas², Marie Claude Bon ${ }^{3}$, \\ Leonardo Marianelli', Iris Bernardinelli ${ }^{4}$, Giorgio Malossini ${ }^{4}$, Luca Benvenuto ${ }^{4}$, \\ Pio Federico Roversi', Kim Hoelmer ${ }^{5}$
}

I CREA - Research Centre for Plant Protection and Certification, Florence, Italy 2 Florida State Collection of Arthropods, Division of Plant Industry, Florida Department of Agriculture and Consumer Services, Gainesville, FL 32608, USA 3 USDA-ARS European Biological Control Laboratory, 810 Avenue du Campus Agropolis, 34980 Montferrier le Lez, France 4 ERSA - Regional Agency for Rural Development, Plant Health Service, Udine, Italy 5 USDA-ARS Beneficial Insects Introduction Research Unit, Newark, DE, USA

Corresponding author: Elijah Talamas (talamas.1@osu.edu)

Academic editor: G. Broad | Received 27 October 2018 | Accepted 4 December 2018 | Published 31 December 2018

http://zoobank.org/2B206556-8118-4E60-AC52-42C827F00B26

Citation: Sabbatini Peverieri G, Talamas E, Bon MC, Marianelli L, Bernardinelli I, Malossini G, Benvenuto L, Roversi PF, Hoelmer K (2018) Two Asian egg parasitoids of Halyomorpha halys (Stål) (Hemiptera, Pentatomidae) emerge in northern Italy: Trissolcus mitsukurii (Ashmead) and Trissolcus japonicus (Ashmead) (Hymenoptera, Scelionidae). Journal of Hymenoptera Research 67: 37-53. https://doi.org/10.3897/jhr.67.30883

\begin{abstract}
Halyomorpha halys (Stål) is a severe agricultural pest that is spreading worldwide from its original distribution in Asia. Egg parasitoids from Asia, which play a key role in the population dynamics of $H$. halys, are following its host along global pathways. We present the first records of Trissolcus mitsukurii in Europe, and of Trissolcus japonicus in Italy. Both discoveries were made in northern Italy, where $H$. halys is widely present and has reached extremely high population densities in some areas. Given the availability of their host, the distributions and populations of these exotic egg parasitoids are expected to expand, even in the absence of human intervention.
\end{abstract}

\section{Keywords}

Egg parasitoid, brown marmorated stink bug, exotic species

Copyright Giuseppino Sabbatini Peverieri et al. This is an open access article distributed under the terms of the Creative Commons Attribution License (CC BY 4.0), which permits unrestricted use, distribution, and reproduction in any medium, provided the original author and source are credited. 


\section{Introduction}

The invasive stink bug, Halyomorpha halys (Stål) (Hemiptera: Pentatomidae), also known as the brown marmorated stink bug, is now a cosmopolitan pest. From its native range in East Asia, it first became established in North America in the mid-1990s (Hoebeke and Carter 2003), followed by Europe in the mid-2000s (Wermelinger et al. 2008), and is now established in South America (Faúndez and Rider 2017). It has been intercepted numerous times in New Zealand but has not yet established a permanent population there (Ormsby 2018). Halyomorpha halys is a highly polyphagous pest, and severe damages are recorded on forest, urban and agricultural trees, and on the fruits and seeds of crops and horticultural plants (Lee et al. 2013, Leskey and Nielsen 2018). Worldwide, the control of $H$. halys currently relies mainly on pesticides (Kuhar and Kamminga 2017). However, because only broad-spectrum pesticides are effective, beneficial insects are also killed and integrated pest management programs are negatively impacted. Thus, much attention has been given to alternative methods for a long-term solution, including biological control. Among natural enemies of $H$. halys, considerable emphasis has been placed on egg parasitoids, which appear to be the most effective enemies in the native Asian range (Yang et al. 2009, Lee 2015). Several studies explored the ability of parasitoids to attack $H$. halys eggs, both in its native distribution and in the newly invaded areas worldwide (Haye et al. 2015, Talamas et al. 2015, Roversi et al. 2016, Herlihy et al. 2016, Abram et al. 2017, Dieckhoff et al. 2017). Presently, only Asian native species of egg parasitoids appear to be promising candidates as biological control agents, primarily Trissolcus japonicus (Ashmead) (Hymenoptera: Scelionidae) (Zhang et al. 2017).

The spread of $H$. halys has also provided an invasion opportunity for its parasitoids. In 2015 T. japonicus, commonly known as the samurai wasp, was detected in the eastern United States (Talamas et al. 2015), followed shortly by discovery of a second, independently established population in the Pacific Northwest (Milnes et al. 2016). In 2017 and again in 2018, T. japonicus was recorded in Switzerland, its first reported recovery in Europe (Stahl et al. 2018). We present here the discovery of two adventive populations of exotic parasitoids, T. mitsukurii (Ashmead) and T. japonicus, that are parasitizing $H$. halys eggs in the wild in northern Italy.

\section{Methods}

\section{Field survey and recovery of Trissolcus mitsukurii in northeastern Italy}

During the 2018 brown marmorated stink bug monitoring campaign in fruit orchards in the Region of Friuli-Venezia-Giulia (northeastern Italy), personnel of ERSA on August 7, and jointly with personnel of CREA on August 8, noted the presence of darkly colored $H$. halys egg masses, which is an indicator of parasitism (see Results for site descriptions). During the surveys, egg masses of other stink bug 
species were also collected when found. A portion of the egg masses collected in the field were reared in climatic chambers $\left(26^{\circ} \mathrm{C}, 65 \% \mathrm{RH}, 16: 8 \mathrm{~L}: \mathrm{D}\right)$ until adult parasitoids emerged. For further study, adults were kept alive in glass tubes and provided with pure honey droplets as food. The remaining field collected egg masses were reared in a laboratory room in Petri dishes until parasitoids emerged and specimens were stored in ethanol for further studies. All emerged specimens were counted, identified to species and sexed.

\section{Field survey and recovery of Trissolcus japonicus in northwestern Italy}

During routine research activities of CREA personnel on July 27 and August 3, 2018, several $H$. halys egg masses with dark coloration were observed on Acer campestris L. trees in a parking lot near Lodi, a site close to the town of Milan (see Results for site description). All collected egg masses were reared in climatic chambers as described previously, and adult parasitoids were kept alive in rearing tubes with pure honey for further studies. Emerged specimens were counted, identified to species and sexed.

Reared specimens of Trissolcus were identified using the key to Palearctic Trissolcus provided in Talamas et al. (2017). Image Z-stacks were taken with a Canon EOS 80D camera attached to an Olympus BX51 compound scope and a Macropod imaging system from Macroscopic Solutions and were rendered using Helicon Focus (Helisoft). Voucher specimens are deposited in the Florida State Collection of Arthropods (Gainesville, FL), the USDA-ARS European Biological Control Laboratory (EBCL) (Montpellier, France), and CREA-DC (Florence, Italy). Collection data associated with a subset of these specimens are deposited in the Hymenoptera Online Database (hol.osu.edu). Morphological terminology follows Mikó et al (2007).

\section{Character annotations}

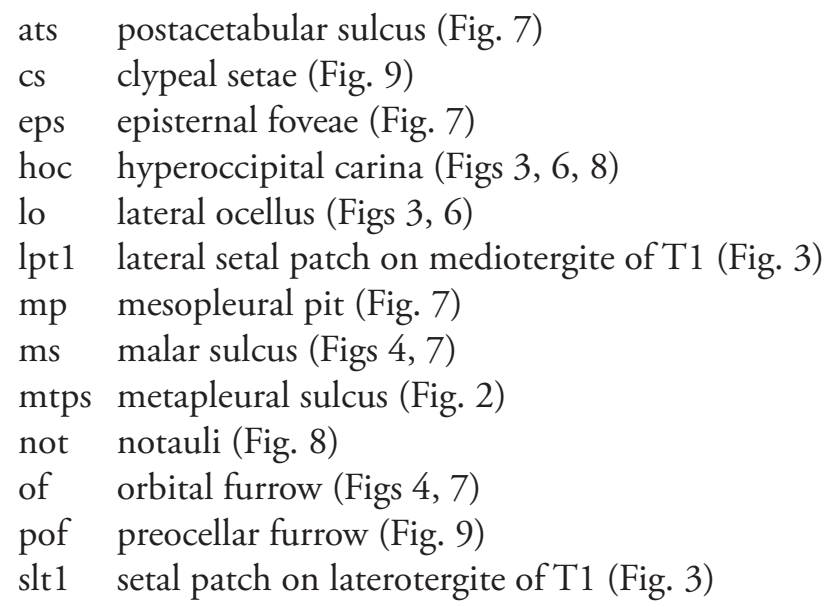




\section{Molecular analysis}

\section{DNA extraction, PCR amplification and sequencing}

Following their morphological examination, all specimens were preserved in $95 \%$ ethanol and shipped to EBCL. Tables 3, 4 list the specimens and voucher information included in the analysis. Prior to DNA extraction, individual specimens were bathed three times at room temperature in molecular grade water for five minutes. Genomic DNA was nondestructively isolated from the entire specimen using the Qiagen DNeasy kit (Hilden, Germany) as published in Taekul et al. (2014) with minor modification. In Step 7, the elution buffer, warmed to $55^{\circ} \mathrm{C}$, was allowed to sit on the membrane during $15 \mathrm{~min}$ before centrifugation. The collected flow-through was reloaded onto the spin column to increase the DNA yield. A negative control (no insect tissue) was included in each extraction to detect potential contamination. The barcode region of the mitochondrial Cytochrome Oxidase Subunit I (COI) was amplified using the universal barcoding primers LCO1490 (5'-GGTCAACAAATCATAAAGATATTGG-3') and HCO2198 (5'-TAAACTTCAGGGTGACCAAAAAATCA-3') (Folmer et al. 1994). Amplification and barcode editing and analysis were done as described in Ganjisaffar et al. (2018). All sequences generated from this study and those from our custom barcode database are deposited in Genbank (Tables 3 and 4) and all residual DNAs are archived at EBCL (Tables 3 and 4). The sequences obtained were compared with sequences present in Genbank by similarity search using the Basic Local Alignment Search Tool (http://www.ncbi.nlm.nih.gov/BLASTn).

Although tracing the source of the Italian populations was not the scope of the present study, we used this barcode approach to provide better insights into the mitochondrial diversity of these two Trissolcus species, identify and exclude Asian populations that were highly divergent from Italian populations, and tentatively find similar populations. Of note, T. mitsukurii barcodes are poorly represented in Genbank database and BOLD as there is only one record published by Mita et al. (2014). For this study we had access to an unpublished EBCL database of 12 barcodes of T. mitsukurii collected in China, Korea and Japan and obtained within the framework of an ongoing USDA biocontrol program. Two sequences generated from the present study were aligned using Clustal W with the sequence from Mita et al. (2014) and the 12 sequences of our custom database.

For T. japonicus, the five sequences generated from this study were aligned with Clustal W with all barcode sequences retrieved from Genbank and BOLD (which contained some sequences not present in Genbank). Only sequences from Asian and Swiss samples were included in the dataset. For both taxa, the phylogenetic relationships among haplotypes were depicted using statistical parsimony in TCS as implemented in PopART (Leigh and Bryant 2015). This approach enabled us to display the geographical distribution of all haplotypes. 


\section{Field surveys}

\section{Trissolcus mitsukurii recoveries in northeastern Italy}

Site descriptions for recovery sites are given in Table 1 . A total of $31 \mathrm{H}$. halys egg masses were collected at three survey sites in northeastern Italy on August 7-8, 2018 (Fig. 1, Table 2). Of these, 4 egg masses were not parasitized, and nymphs hatched at a rate of $98.23 \% ; 25$ egg masses were parasitized by T. mitsukurii; and 2 egg masses were parasitized by Anastatus bifasciatus (Geoffroy) (Eupelmidae) and a species of Pteromalidae. From the 25 egg masses parasitized by T. mitsukurii, a total of 424 adults emerged of which $91.75 \%$ were females. Depending on locality, the parasitism rate of eggs within single egg masses ranged from $32.14 \%$ to $100 \%$ and number of parasitized egg masses out of the total number of egg masses detected ranged among sites from $50.00 \%$ to $84.21 \%$.

During surveys at the Codroipo site, an egg mass of a predatory stink bug belonging to the subfamily of Asopinae was collected which was also parasitized by $T$. mitsukurii. From this egg mass, $83.87 \%$ of the eggs (31 eggs in total) were parasitized (emergence rate $96.15 \% ; 80.00 \%$ females); only one egg of the cluster produced a stink bug nymph, and four apparently unparasitized eggs did not hatch.

\section{Trissolcus japonicus findings in northwestern Italy}

The recovery site of T. japonicus in northwestern Italy was located at $45.3031 \mathrm{~N}$, 9.4794E (Fig. 1). Parasitized egg masses were found on Acer campestris L. trees in a parking lot surrounded by a multi-host patchy landscape (crop fields, uncultivated fields, hedgerows), and industrial and urban areas with ornamental plants.

Table I. Descriptions of Trissolcus mitsukurii recovery sites in northeastern Italy.

\begin{tabular}{|c|c|c|c|c|}
\hline Locality & Coordinates & $\begin{array}{l}\text { Main culture } \\
\text { (plant species and } \\
\text { management) }\end{array}$ & Surrouding cultures & Surrounding environment \\
\hline Cordenons site 1 & $\begin{array}{l}46.0089 \mathrm{~N} \\
12.6824 \mathrm{E}\end{array}$ & $\begin{array}{l}\text { Kiwi orchard } \\
\text { (Actinidia chinensis), } \\
\text { organic farming }\end{array}$ & $\begin{array}{l}\text { Vineyards, maize and soybean } \\
\text { crops managed by integrated } \\
\text { pest management }\end{array}$ & $\begin{array}{c}\text { Hedgerows, apple and kiwi } \\
\text { orchards, vineyards, maize } \\
\text { and soybean fields }\end{array}$ \\
\hline Cordenons site 2 & $\begin{array}{l}46.0082 \mathrm{~N} \\
12.6713 \mathrm{E}\end{array}$ & $\begin{array}{c}\text { Hedgerow (Robinia } \\
\text { pseudoacacia) }\end{array}$ & $\begin{array}{l}\text { Apple orchard, vineyards, maize } \\
\text { and soybean crops managed by } \\
\text { integrated pest management }\end{array}$ & $\begin{array}{c}\text { Hedgerows, apple and kiwi } \\
\text { orchards, vineyards, maize } \\
\text { and soybean fields }\end{array}$ \\
\hline Codroipo & $\begin{array}{c}45.9675 \mathrm{~N} \\
13.0251 \mathrm{E}\end{array}$ & $\begin{array}{l}\text { Kiwi orchard } \\
\text { (Actinidia deliciosa), } \\
\text { integrated pest } \\
\text { management }\end{array}$ & $\begin{array}{c}\text { Apple and pear orchards, } \\
\text { vineyards, maize and soybean } \\
\text { crops managed by integrated } \\
\text { pest management }\end{array}$ & $\begin{array}{c}\text { Hedgerows, apple and pear } \\
\text { orchards, vineyards, maize } \\
\text { and soybean fields }\end{array}$ \\
\hline
\end{tabular}




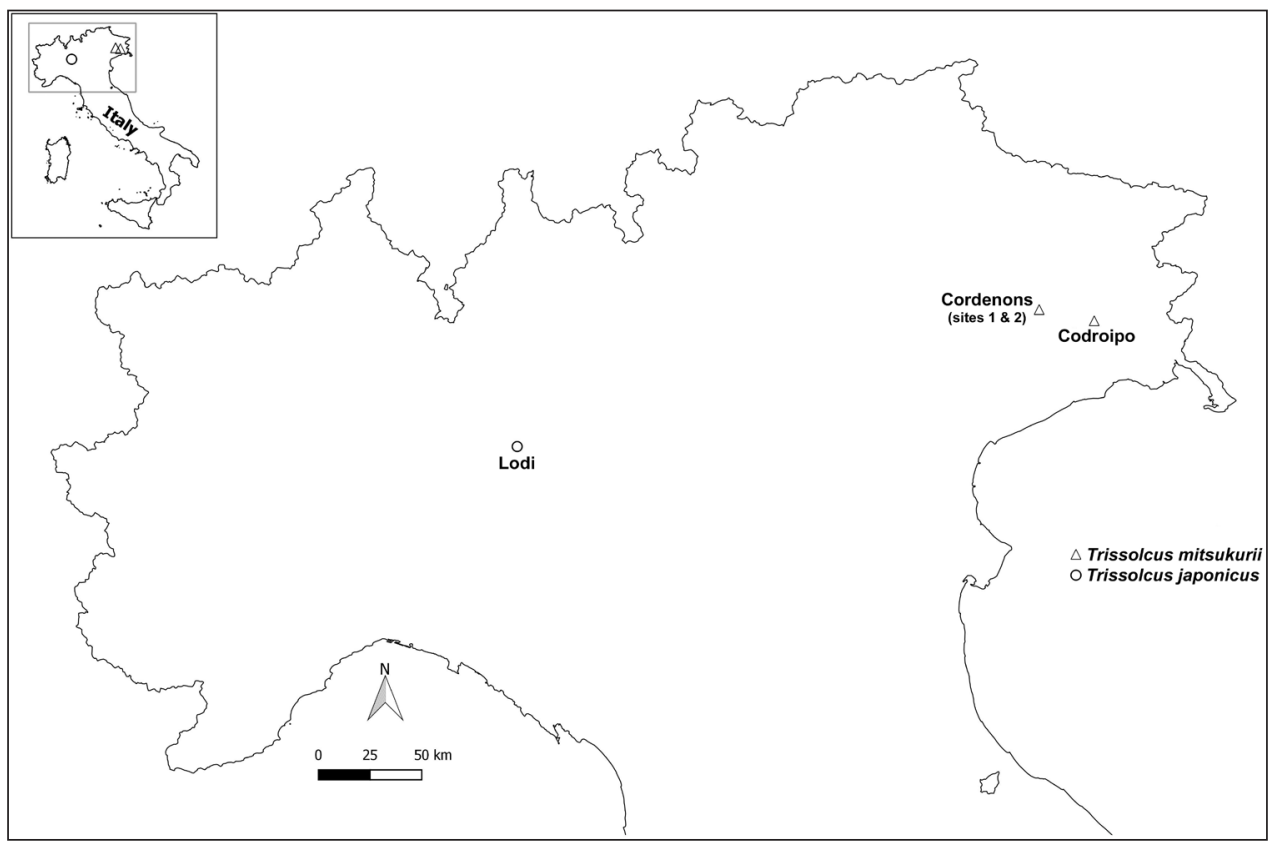

Figure I. Recovery sites of Trissolcus mitsukurii and Trissolcus japonicus in northern Italy.

Table 2. Parasitism of Halyomorpha halys eggs by Trissolcus mitsukurii collected in field surveys of August 7-8, 2018, in northeastern Italy.

\begin{tabular}{|c|c|c|c|c|c|c|c|}
\hline \multirow[b]{2}{*}{ Site } & \multirow{2}{*}{ 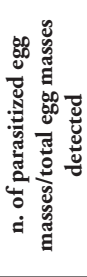 } & \multirow{2}{*}{ 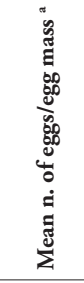 } & \multicolumn{4}{|c|}{ Parasitized egg masses } & \multirow{2}{*}{ 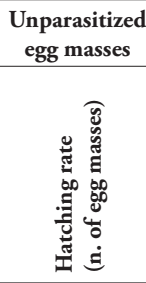 } \\
\hline & & & 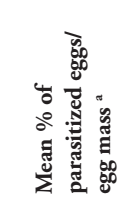 & 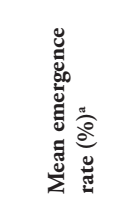 & 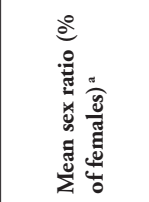 & 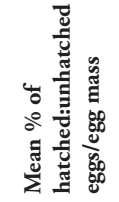 & \\
\hline \multirow{2}{*}{ Cordenons site 1} & \multirow{2}{*}{$16: 19$} & 28.00 & 87.04 & 94.22 & 92.31 & \multirow{2}{*}{$0.00: 12.74$} & \multirow{2}{*}{$100 \%(3)$} \\
\hline & & $(27-29)$ & $(32.14-100)$ & $(57.89-100)$ & $(88.99-100)$ & & \\
\hline \multirow{2}{*}{ Cordenons site 2} & \multirow{2}{*}{$1: 2^{*}$} & 21.50 & \multirow{2}{*}{100} & \multirow{2}{*}{100} & \multirow{2}{*}{92.86} & \multirow{2}{*}{$0.00: 0.00$} & \multirow{2}{*}{ not assessed $(0)$} \\
\hline & & $(15-28)$ & & & & & \\
\hline \multirow{2}{*}{ Codroipo } & \multirow{2}{*}{$8: 10^{*}$} & 28.00 & 92.89 & 90.30 & 90.44 & \multirow{2}{*}{$0.00: 3.59$} & \multirow{2}{*}{$100 \%(1)$} \\
\hline & & $(27-29)$ & $(78.57-100)$ & $(65.38-100)$ & $(84.21-95.45)$ & & \\
\hline
\end{tabular}

* one egg mass was entirely parasitized by other parasitoids. ${ }^{a}$ minimum and maximum values in parentheses

Field collections were made on July 27 and August 3, 2018, and a total of $45 H$. halys egg masses were collected (with a mean of 25.77 eggs/egg mass). On July 27, only one egg mass was detected and was found to be successfully parasitized by T. japonicus, with the emergence of 8 specimens, all of which were males. Among the 44 egg masses collected on August 3, 21 egg masses were not parasitized and eggs hatched into nymphs with a mean rate of $82.44 \%$, and 22 egg masses were parasitized by $A$. bifasciatus. Only 
Table 3. Sampling Information, GenBank Accession numbers and haplotypes for Trissolcus mitsukurii included in this study.

\begin{tabular}{|c|c|c|c|c|c|c|c|}
\hline Collection code and Sex & Country & Site & $\begin{array}{l}\text { Year of Collection, } \\
\text { Name of Collector }{ }^{b}\end{array}$ & Host & $\begin{array}{c}\text { GenBank } \\
\text { Accession } \\
\text { Number }\end{array}$ & $\begin{array}{c}\text { Barcode } \\
\text { Haplotype } \\
\text { (617bp) }\end{array}$ & $\begin{array}{r}\text { Isolate } \\
\text { (EBCL) }\end{array}$ \\
\hline FSCA00033071, ㅇ & Italy & $\begin{array}{c}\text { Cordenons, } \\
\text { Friuli-Venezia- } \\
\text { Giulia }\end{array}$ & 2018, IB, LB \& GM & Halyomorpha halys & $\begin{array}{l}\text { MK097189 } \\
\text { (this study) }\end{array}$ & $\mathrm{H} 5$ & Tsp270 \\
\hline FSCA00033072, 우 & Italy & $\begin{array}{l}\text { Cordenons, } \\
\text { Friuli-Venezia- } \\
\text { Giulia }\end{array}$ & 2018, IB, LB \& GM & H. halys & $\begin{array}{l}\text { MK097190 } \\
\text { (this study) }\end{array}$ & $\mathrm{H} 5$ & Tsp269 \\
\hline Tm1-EBCL ${ }^{\mathrm{a}},{ }^{\lambda}$ & Japan & $\begin{array}{l}\text { Tsukuba, } \\
(\mathrm{NARO})^{c}\end{array}$ & 2007, KH & H. halys & $\begin{array}{l}\text { MK097191 } \\
\text { (this study) }\end{array}$ & H1 & $\operatorname{Tm} 1$ \\
\hline USNMENT01197989, ㅇ & $\begin{array}{l}\text { South } \\
\text { Korea }\end{array}$ & Gochang & $2015, \mathrm{KH}$ & $\begin{array}{l}\text { unidentified host } \\
\text { eggs (not } H \text {. halys) }\end{array}$ & $\begin{array}{l}\text { MK097192 } \\
\text { (this study) }\end{array}$ & $\mathrm{H} 3$ & Tsp202 \\
\hline USNMENT01197242, ㅇ & $\begin{array}{l}\text { South } \\
\text { Korea }\end{array}$ & Jeju & 2012, ET\& IM & na & $\begin{array}{l}\text { MK097193 } \\
\text { (this study) }\end{array}$ & $\mathrm{H} 4$ & Tsp233 \\
\hline USNMENT01197243, ㅇ & $\begin{array}{l}\text { South } \\
\text { Korea }\end{array}$ & Jeju & 2012, ET\& IM & na & $\begin{array}{l}\text { MK097194 } \\
\text { (this study) }\end{array}$ & H4 & Tsp234 \\
\hline USNMENT01197244, ㅇ & $\begin{array}{l}\text { South } \\
\text { Korea }\end{array}$ & Jeju & 2012, ET\& IM & na & $\begin{array}{l}\text { MK097195 } \\
\text { (this study) }\end{array}$ & $\mathrm{H} 4$ & Tsp235 \\
\hline USNMENT00977533, 우 & China & $\begin{array}{l}\text { Yunnan Prov., } \\
\text { Kunming }\end{array}$ & 2013, KH & Erthesina fullo & $\begin{array}{l}\text { MK097196 } \\
\text { (this study) }\end{array}$ & $\mathrm{H} 2$ & Tsp39 \\
\hline USNMENT01059335, 우 & China & $\begin{array}{l}\text { Yunnan Prov., } \\
\text { Kunming }\end{array}$ & $2013, \mathrm{KH}$ & E. fullo & $\begin{array}{l}\text { MK097197 } \\
\text { (this study) }\end{array}$ & $\mathrm{H} 2$ & Tsp60 \\
\hline USNMENT01197294, + & Japan & $\begin{array}{l}\text { Tsukuba, } \\
\text { (NARO) }^{c}\end{array}$ & $2012, \mathrm{KH}$ & H. halys & $\begin{array}{l}\text { MK097198 } \\
\text { (this study) }\end{array}$ & $\mathrm{H} 1$ & Tsp149 \\
\hline USNMENT01197295, ㅇ & Japan & $\begin{array}{l}\text { Tsukuba, } \\
\text { (NARO) }^{c}\end{array}$ & 2012, KH & H. halys & $\begin{array}{l}\text { MK097199 } \\
\text { (this study) }\end{array}$ & $\mathrm{H} 1$ & Tsp150 \\
\hline Tsp151- EBCL ${ }^{a}$, na & Japan & $\begin{array}{l}\text { Tsukuba, } \\
\text { (NARO) }^{c}\end{array}$ & 2012, KH & H. halys & $\begin{array}{l}\text { MK097200 } \\
\text { (this study) }\end{array}$ & H1 & Tsp151 \\
\hline Tsp152-EBCLa, na & Japan & $\begin{array}{l}\text { Tsukuba, } \\
\text { (NARO) }^{c}\end{array}$ & $2012, \mathrm{KH}$ & H. halys & $\begin{array}{l}\text { MK097201 } \\
\text { (this study) }\end{array}$ & $\mathrm{H} 1$ & Tsp152 \\
\hline Tsp153- EBCL ${ }^{\mathrm{a}}$, na & Japan & $\begin{array}{l}\text { Tsukuba, } \\
\text { (NARO) }^{c}\end{array}$ & 2012, KH & H. halys & $\begin{array}{l}\text { MK097202 } \\
\text { (this study) }\end{array}$ & H1 & Tsp153 \\
\hline na, na & Japan & Fukuoka & na, na & Nezara viridula & $\begin{array}{c}\text { AB971831 } \\
\text { (Mita et al. } \\
\text { 2014) }\end{array}$ & H1 & \\
\hline
\end{tabular}

${ }^{a}$ EBCL DNA collection. ${ }^{b}$ name of collectors: IB: Iris Bernardinelli, LB: Luca Benvenuto, GM: Giorgio Malossini, KH: Kim Hoelmer, ET: Elijah Talamas, IM: Istvan Mikó. ${ }^{\mathrm{c}}$ NARO: National Agriculture and Food Research Organization.

one egg mass collected on August 3 was parasitized by T. japonicus. All eggs that were apparently parasitized (35.71\% of eggs in the mass) produced adult parasitoids ( 9 females and 2 males), $46.43 \%$ of eggs were unhatched and $17.86 \%$ of the eggs hatched into stink bug nymphs. Ninety percent of the emerged T. japonicus were females.

\section{Taxonomy}

\section{Trissolcus mitsukurii}

Trissolcus mitsukurii is a straightforward species to identify and is separated early in the key to Palearctic Trissolcus (Talamas et al. 2017). We thus do not consider it necessary to produce an updated key to European Trissolcus that includes this species, and instead 
Table 4. Sampling Information, GenBank Accession numbers and haplotype for Trissolcus japonicus included in this study.

\begin{tabular}{|c|c|c|c|c|c|c|c|}
\hline $\begin{array}{l}\text { Collection code and } \\
\text { Sex }\end{array}$ & Country & Site & $\begin{array}{c}\text { Year of } \\
\text { Collection, } \\
\text { Name of } \\
\text { Collector }^{b}\end{array}$ & Host & $\begin{array}{l}\text { GenBank Accession Number/ } \\
\text { Bold Accession number }\end{array}$ & $\begin{array}{l}\text { Barcode } \\
\text { Haplotype } \\
\text { (373bp) }\end{array}$ & $\begin{array}{c}\text { Isolate } \\
\text { (EBCL) }\end{array}$ \\
\hline FSCA $00033060, q$ & Italy & $\begin{array}{l}\text { Lodi, } \\
\text { Lombardy }\end{array}$ & 2018, PFR & $\begin{array}{l}\text { Halyomorpha } \\
\text { halys }\end{array}$ & MK097184 (this study) & H1 & $\mathrm{Tj} 406$ \\
\hline FSCA 00033065 , ㅇ & Italy & $\begin{array}{c}\text { Lodi, } \\
\text { Lombardy }\end{array}$ & 2018, PFR & H. halys & MK097185 (this study) & $\mathrm{H} 1$ & $\mathrm{Tj} 408$ \\
\hline FSCA0033097, ठ & Italy & $\begin{array}{l}\text { Lodi, } \\
\text { Lombardy }\end{array}$ & 2018, PFR & H. halys & MK097186 (this study) & H1 & $\mathrm{Tj} 421$ \\
\hline FSCA0033098, $\widehat{\partial}$ & Italy & $\begin{array}{l}\text { Lodi, } \\
\text { Lombardy }\end{array}$ & 2018, PFR & H. halys & MK097187 (this study) & $\mathrm{H} 1$ & $\mathrm{Tj} 422$ \\
\hline FSCA0033096, ठิ & Italy & $\begin{array}{c}\text { Lodi, } \\
\text { Lombardy }\end{array}$ & 2018, PFR & H. halys & MK097188 (this study) & H1 & Тј423 \\
\hline GBIFCH00543446, 우 & Switzerland & Ticino & 2017, JS & H. halys & MH919753 (Stahl et al., 2018) & H1 & Tj388 \\
\hline GBIFCH00543447, 우 & Switzerland & Ticino & 2017, JS & H. halys & MH919754 (Stahl et al., 2018) & $\mathrm{H} 1$ & Tj389 \\
\hline GBIFCH00543448, 우 & Switzerland & Ticino & 2017, JS & H. halys & MH919755 (Stahl et al., 2018) & $\mathrm{H} 1$ & Tj390 \\
\hline GBIFCH00543449, ठ̊ & Switzerland & Ticino & 2017, JS & H. halys & MH919756 (Stahl et al., 2018) & H1 & Tj391 \\
\hline GBIFCH00543450, ठ̊ & Switzerland & Ticino & 2017, JS & H. halys & MH919757 (Stahl et al., 2018) & $\mathrm{H} 1$ & Tj392 \\
\hline GBIFCH00543451, ठ̊ & Switzerland & Ticino & 2017, JS & H. halys & MH919758 (Stahl et al., 2018) & $\mathrm{H} 1$ & Tj393 \\
\hline Tsp77- EBCLaa, na & Japan & $\begin{array}{l}\text { Tsukuba } \\
(\mathrm{NARO})^{\mathrm{c}}\end{array}$ & 2012, KH & H. halys & $\begin{array}{c}\text { MH919744 (Bon et al., } \\
\text { unpublished) }\end{array}$ & $\mathrm{H} 1$ & Tsp77 \\
\hline Tsp78- EBCLaa, na & Japan & $\begin{array}{l}\text { Tsukuba } \\
(\mathrm{NARO})^{\mathrm{c}}\end{array}$ & 2012, KH & H. halys & $\begin{array}{l}\text { MH919745 (Bon et al., } \\
\text { unpublished) }\end{array}$ & H1 & Tsp78 \\
\hline Tsp79- EBCLa , na & Japan & $\begin{array}{l}\text { Tsukuba } \\
\text { (NARO) }^{c}\end{array}$ & 2012, KH & H. halys & $\begin{array}{l}\text { MH919746 (Bon et al., } \\
\text { unpublished) }\end{array}$ & H1 & Tsp79 \\
\hline Tsp88- EBCL ${ }^{2}$, na & Japan & $\begin{array}{l}\text { Tsukuba } \\
\text { (NARO) }^{c}\end{array}$ & 2012, KH & H. halys & $\begin{array}{l}\text { MH919747 (Bon et al., } \\
\text { unpublished) }\end{array}$ & H1 & Tsp88 \\
\hline Tsp90-EBCL ${ }^{a}$, na & Japan & $\begin{array}{l}\text { Tsukuba } \\
\text { (NARO) }^{c}\end{array}$ & 2012, KH & H. halys & $\begin{array}{l}\text { MH919748 (Bon et al., } \\
\text { unpublished) }\end{array}$ & H1 & Tsp90 \\
\hline Tsp91-EBCL ${ }^{\mathrm{a}}$, na & Japan & $\begin{array}{l}\text { Tsukuba } \\
\text { (NARO) }^{c}\end{array}$ & 2012, KH & H. halys & $\begin{array}{l}\text { MH919749 (Bon et al., } \\
\text { unpublished) }\end{array}$ & H1 & Tsp91 \\
\hline Tsp93- EBCL a , na & Japan & $\begin{array}{l}\text { Tsukuba } \\
(\mathrm{NARO})^{c}\end{array}$ & 2012, KH & H. halys & $\begin{array}{l}\text { MH919750 (Bon et al., } \\
\text { unpublished) }\end{array}$ & $\mathrm{H} 1$ & Tsp93 \\
\hline Tsp226-EBCL & Japan & Kanagawa & 2015, KH & Plautia stali & $\begin{array}{l}\text { MH919752 (Bon et al., } \\
\text { unpublished) }\end{array}$ & H1 & Tsp226 \\
\hline na, $q$ & Japan & Kanagawa & 2012, TM & P. stali & $\begin{array}{l}\text { AB847131-32,36 } \\
\text { (Matsuo et al., 2014) }\end{array}$ & H1 & \\
\hline $\mathrm{na},+$ & Japan & Fukuoka & 2012, KM & P. stali & $\begin{array}{c}\text { AB847144-145 } \\
\text { (Matsuo et al., 2014) }\end{array}$ & $\mathrm{H} 2$ & \\
\hline na, & Japan & Fukuoka & 2012, KM & H. halys & $\begin{array}{c}\text { AB908179-182 } \\
\text { (Matsuo et al., 2014) }\end{array}$ & $\mathrm{H} 2$ & \\
\hline na, na & Japan & na & na, na & na & $\begin{array}{l}\text { AB894834-35, AB894838-39 } \\
\text { (Matsuo, K. and Hirose,Y., } \\
\text { unpublished }\end{array}$ & $\mathrm{H} 2$ & \\
\hline na, $q$ & Japan & Fukuoka & 2012, KM & P. stali & $\begin{array}{l}\text { AB847129,130, 137,143,146 } \\
\text { (Matsuo et al., 2014) }\end{array}$ & $\mathrm{H} 3$ & \\
\hline na, na & Japan & na & na, na & na & $\begin{array}{l}\text { AB894836,837,840,841 } \\
\text { (Matsuo, K. \& Hirose,Y. } \\
\text { (unpublished), }\end{array}$ & $\mathrm{H} 3$ & \\
\hline na, na & Japan & Kanagawa & na, na & P. stali & AB971832 (Mita et al., 2014) & $\mathrm{H} 1$ & \\
\hline na, na & China & & & H. halys & $\begin{array}{c}\text { KF303518.1 } \\
\text { (Gariepy et al., 2014) }\end{array}$ & $\mathrm{H} 7$ & \\
\hline $\begin{array}{l}\text { USNMENT01059340, } \\
+\underset{+}{ }\end{array}$ & China & Langfang & 2012, KH & E. fullo & $\begin{array}{c}\text { / NSCEL009-18 } \\
\text { (Gariepy unpublished) }\end{array}$ & $\mathrm{H} 4$ & Tsp61 \\
\hline $\begin{array}{l}\text { USNMENT01197300, } \\
\text { q }\end{array}$ & China & Kunming & 2014, KH & E. fullo & $\begin{array}{l}\text { /NSCEL010-18 } \\
\text { Gariepy unpublished) }\end{array}$ & H5 & Tsp155 \\
\hline Tsp1-EBCL ${ }^{\text {a }}$, na & Japan & $\begin{array}{l}\text { Tsukuba } \\
\text { (NARO) }^{c}\end{array}$ & 2012, KH & H. halys & /NSCEL011-18 & $\mathrm{H} 1$ & Tsp1 \\
\hline
\end{tabular}




\begin{tabular}{|c|c|c|c|c|c|c|c|}
\hline $\begin{array}{l}\text { Collection code and } \\
\text { Sex }\end{array}$ & Country & Site & $\begin{array}{c}\text { Year of } \\
\text { Collection, } \\
\text { Name of } \\
\text { Collector }^{b}\end{array}$ & Host & $\begin{array}{l}\text { GenBank Accession Number/ } \\
\text { Bold Accession number }\end{array}$ & $\begin{array}{c}\text { Barcode } \\
\text { Haplotype } \\
\text { (373bp) }\end{array}$ & $\begin{array}{c}\text { Isolate } \\
\text { (EBCL) }\end{array}$ \\
\hline $\begin{array}{l}\text { USNMENT00977534, } \\
\text { q }\end{array}$ & S. Korea & $\begin{array}{l}\text { Jirisan } \\
\text { Park }\end{array}$ & 2013, KH & H. halys & $\begin{array}{l}\text { /NSCEL012-18 } \\
\text { Gariepy unpublished) }\end{array}$ & H6 & Tsp53 \\
\hline Tj1-EBCL ${ }^{a}$, na & China & Hebei & $2012, \mathrm{KH}$ & H. halys & $\begin{array}{l}\text { /NSCEL013-18 } \\
\text { Gariepy unpublished) }\end{array}$ & $\mathrm{H} 7$ & $\mathrm{Tj} 1$ \\
\hline Trj2-EBCL ${ }^{a}$, na & China & Hebei & 2012, KH & H. halys & $\begin{array}{l}\text { /NSCEL014-18 } \\
\text { Gariepy unpublished) }\end{array}$ & $\mathrm{H} 7$ & $\operatorname{Trj} 2$ \\
\hline $\begin{array}{l}\text { USNMENT01197806, } \\
\text { q }\end{array}$ & Japan & Kanagawa & 2015, KH & H. halys & $\begin{array}{l}\text { /NSCEL017-18 } \\
\text { Gariepy unpublished) }\end{array}$ & H1 & Tsp223 \\
\hline $\begin{array}{l}\text { USNMENT01197320, } \\
\text { + }\end{array}$ & $\begin{array}{l}\text { South } \\
\text { Korea }\end{array}$ & Seoul & 2014, KH & H. halys & $\begin{array}{l}\text { /NSCEL018-18 } \\
\text { Gariepy unpublished) }\end{array}$ & $\mathrm{H} 7$ & Tsp175 \\
\hline na, na & China & Hebei & 2012, TH & H. halys & $\begin{array}{l}\text { /PPENT028-12 } \\
\text { Gariepy unpublished) }\end{array}$ & $\mathrm{H} 7$ & \\
\hline na, na & China & Hebei & 2012, TH & H. halys & $\begin{array}{l}\text { /PPENT029-12 } \\
\text { Gariepy unpublished) }\end{array}$ & $\mathrm{H} 7$ & \\
\hline na, na & China & Hebei & $2012, \mathrm{TH}$ & H. halys & $\begin{array}{c}\text { /PPENT030-12 } \\
\text { Gariepy unpublished) }\end{array}$ & H7 & \\
\hline na, na & China & Hebei & 2012, TH & H. halys & $\begin{array}{l}\text { /PPENT031-12 } \\
\text { Gariepy unpublished) }\end{array}$ & $\mathrm{H} 7$ & \\
\hline na, na & China & Hebei & 2012, TH & H. halys & $\begin{array}{l}\text { /PPENT032-12 } \\
\text { Gariepy unpublished) }\end{array}$ & $\mathrm{H} 7$ & \\
\hline
\end{tabular}

${ }^{a}$ EBCL DNA collection. ${ }^{b}$ name of collector: PFR: Pio Federico Roversi, JS: Judith Stahl, KH: Kim Hoelmer, KM: Kazunori Matsuo, TM: Toshiharu Mita, TH: Tim Haye. ${ }^{c}$ NARO: National Agriculture and Food Research Organization.

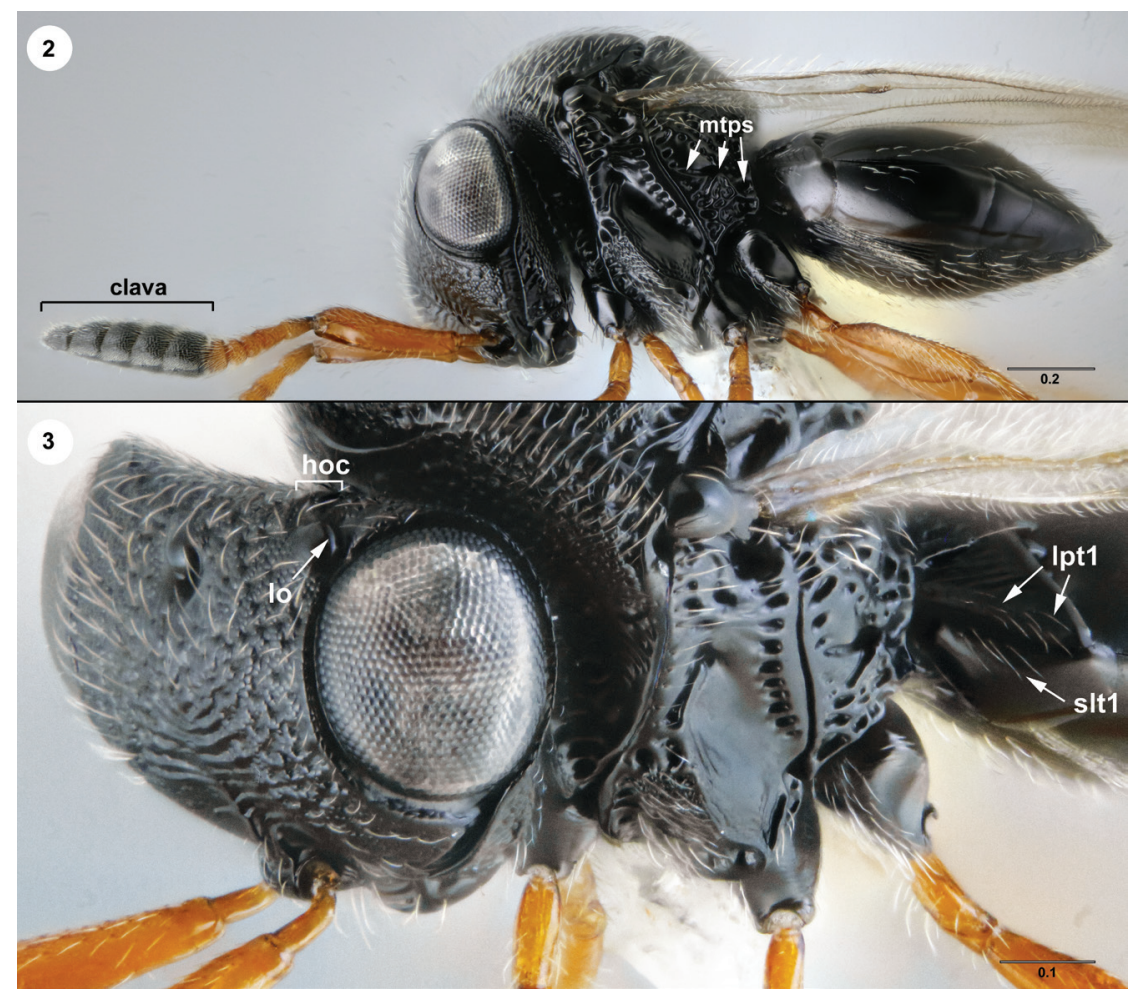

Figures 2-3. Trissolcus mitsukurii, female (FSCA 00033025) 2 head, mesosoma, metasoma, lateral view 3 head, mesosoma, metasoma, anterolateral view. Scale bars in millimeters. 


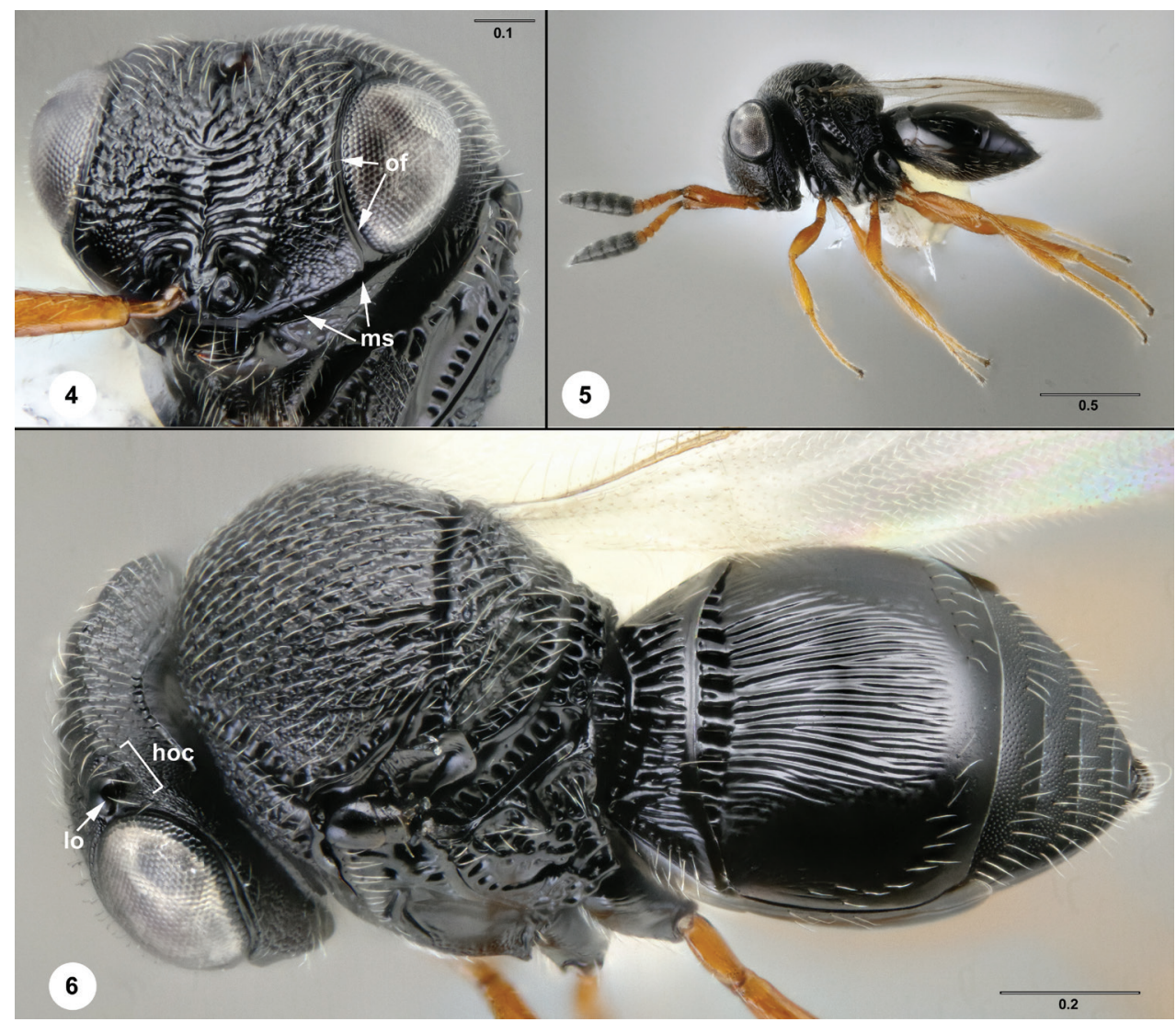

Figures 4-6. Trissolcus mitsukurii, female (FSCA 00033025) $\mathbf{4}$ head, anterior view $\mathbf{5}$ habitus, lateral view 6 head, mesosoma, metasoma, dorsolateral view. Scale bars in millimeters.

we provide the following diagnosis, with references to illustrations, that will be helpful for distinguishing T. mitsukurii from the European fauna: the clava of the female antenna is 5-merous, and the clavomeres are distinctly larger and darker than preceding antennomeres (Fig. 2); the orbital furrow is expanded where it intersects the malar sulcus (Fig. 4); the hyperoccipital carina is often present directly posterior to the lateral ocellus, but is absent between the ocelli (Figs 3, 6); the metapleuron is without setae below the metapleural sulcus (Fig. 3); a setal patch is present on the first laterotergite of the metasoma (slt1, Fig. 3). Care should be taken not to confuse the setal patch of the laterotergite with the setal patch on the mediotergite (lpt1, Fig. 3).

\section{Trissolcus japonicus}

The key to European Trissolcus in Talamas et al. (2017) included T. japonicus to provide identification of this species given the possibility that it might become estab- 


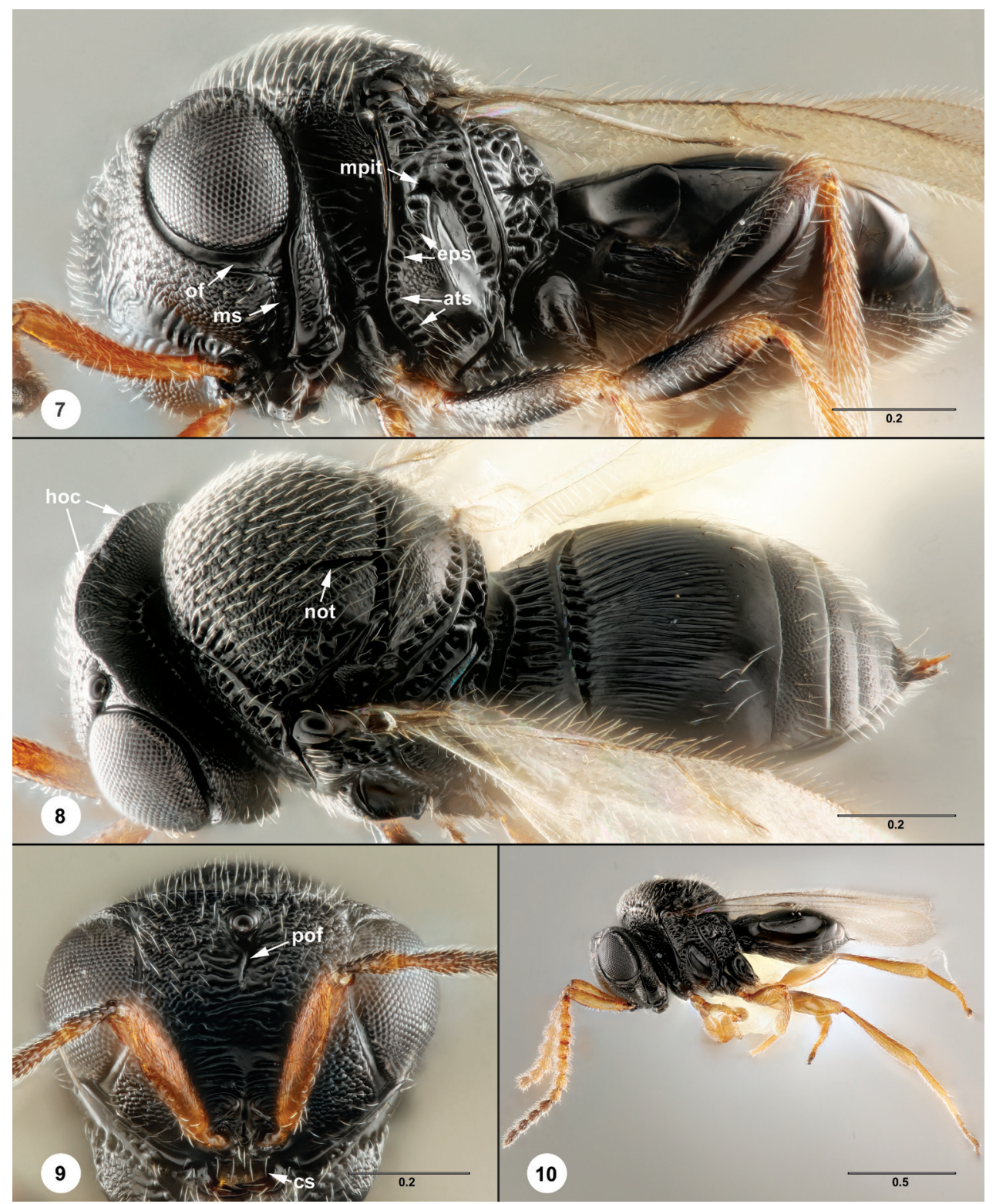

Figures 7-10. Trissolcus japonicus $\mathbf{7}$ female (FSCA 00033063), head, mesosoma, metasoma, ventrolateral view 8 female (FSCA 00033063), head, mesosoma, metasoma, dorsolateral view 9 female (FSCA 00033063) head, anterior view 10 male (FSCA 00033095), habitus, lateral view. Scale bars in millimeters.

lished in Europe. The specimens from northern Italy are fully congruent with the concept of this species presented by Talamas et al. (2017). Trissolcus japonicus can be separated from other species of European Trissolcus by the following diagnosis: four clypeal setae are present below the antennal insertions (Fig. 9); microsculpture is pre- 
sent throughout the frons (Fig. 9); the orbital furrow is expanded at its intersection with the malar sulcus (Fig. 7); the hyperoccipital carina is complete (Fig. 8); episternal foveae extend from the postacetabular sulcus to the mesopleural pit (Fig. 7); and the mesoscutum is without oblique rugae between the notauli (Fig. 8). The preocellar furrow, which extends ventrally from the median ocellus (Fig. 9), is a useful character for confirming the identity of T. japonicus, but it is not always present, and exhibits the greatest variability in males.

\section{Anastatus bifasciatus}

Specimens of $A$. bifasciatus were identified by GSP using the keys of Kalina (1981) and Askew and Nieves-Aldrey (2014) and the identification was confirmed by Dr. Lucian Fusu (University of Iasi, Romania) who compared them with authoritatively identified specimens.

\section{Molecular identification}

\section{Trissolcus mitsukurii}

The two voucher specimens recovered from the field in Cordenons (site 1) in the region of Friuli-Venezia-Giula yielded a similar barcode sequence of 666-bp in length. A BLAST search showed the best similarity score (99\%) of this barcode sequence with T. mitsukurii (Accession No. AB971831). From the final alignment of 617-bp of 15 T. mitsukurii barcodes, a total of five haplotypes (denoted H1-H5) were recovered (Table 3). The haplotype $\mathrm{H} 5$ found in Italy is a new haplotype, as it did not match any haplotype found so far in Asia (Fig. 11, Table 3). From the network analysis, H5 differed by seven substitutions from the two closest haplotypes $\mathrm{H} 1$ and $\mathrm{H} 2$ from Japan and China respectively (Fig. 11).

\section{Trissolcus japonicus}

The five voucher specimens recovered from the field in Lodi yielded a unique barcode sequence of 666-bp in length. A BLAST search showed the best similarity score (100\%) of this barcode sequence with T. japonicus (Accession No. AB971832). From the final alignment of 373-bp of 60 T. japonicus barcodes, a total of seven haplotypes (denoted $\mathrm{H} 1$ to H7) were recovered (Table 4). The haplotype H1 found in Italy was also found in Tsukuba and Kanagawa in Japan, and in Switzerland (Fig. 12, Table 4). H1 is also the predominant haplotype found in the specimens collected in Japan (45\%). Samples from China were the most diverse, displaying 4 haplotypes (H4, H5, H6, H7), although they represent only $22 \%$ of the total sampling. From the network analysis, H7 from China and South Korea differed only by one substitution from the haplotype 


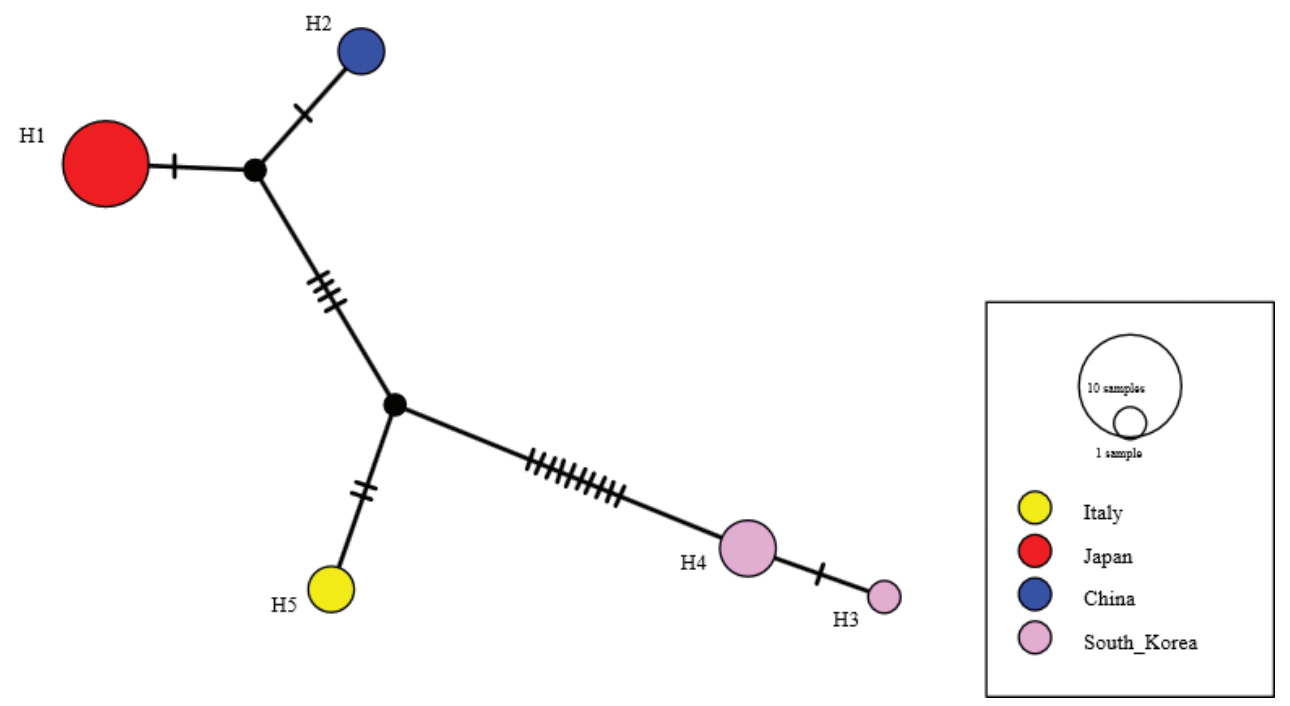

Figure II. COI haplotype network of the Trissolcus mitsukurii analyzed in this study. Each circle corresponds to one haplotype; circle size gives the proportion of individuals belonging to the haplotype. The color inside each circle represents the geographical origin. Numbers correspond to the haplotype numbers. Hatch marks symbolize the number of mutations between haplotypes.

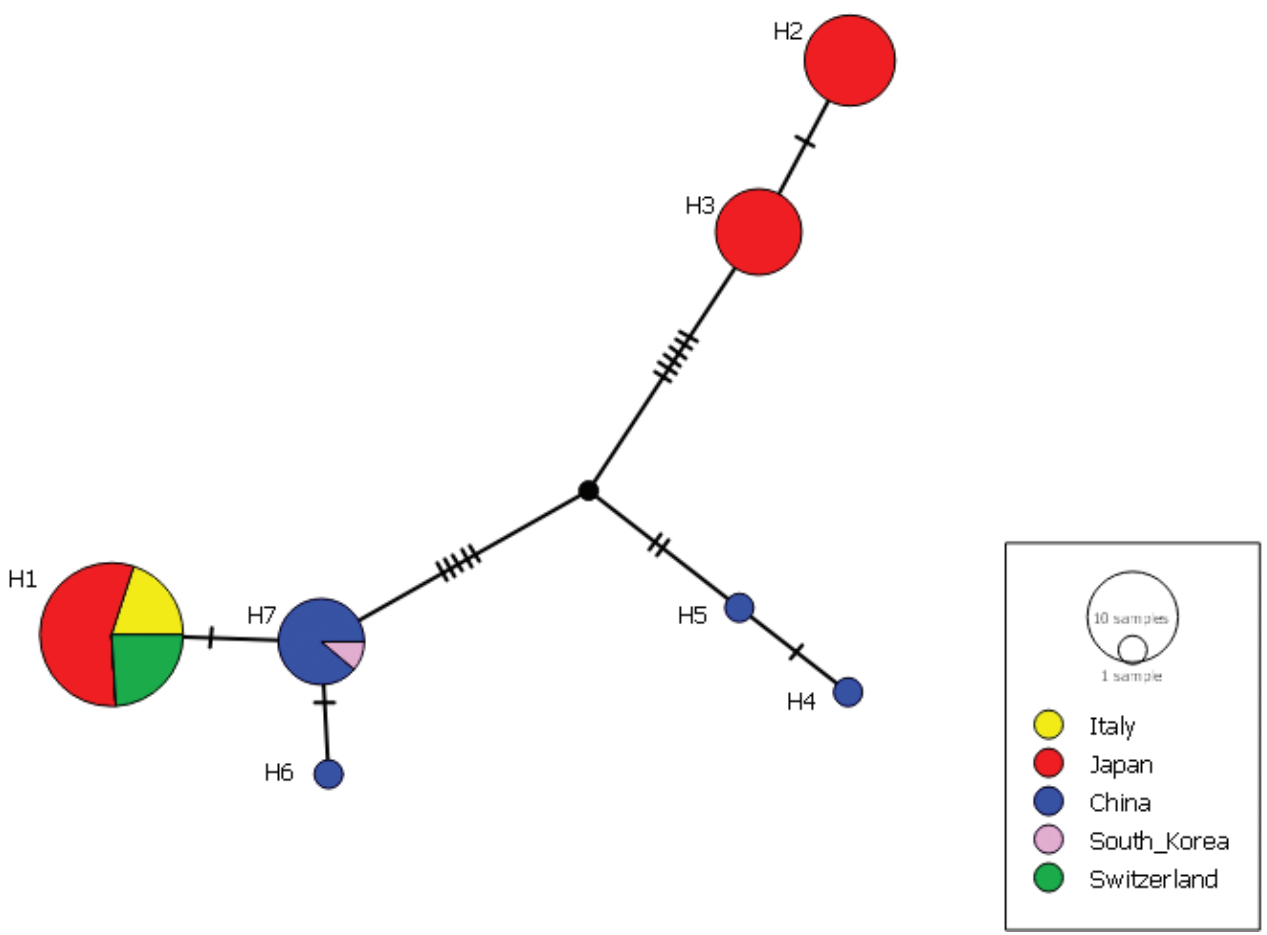

Figure 12. COI haplotype network of the Trissolcus japonicus analyzed in this study. Each circle corresponds to one haplotype; circle size gives the proportion of individuals belonging to the haplotype. The color inside each circle represents the geographical origin. Numbers correspond to the haplotype numbers. Hatch marks symbolise the number of mutations between haplotypes. 
H1 (Fig. 12). Although our haplotype analysis evidenced a best match of the Italian populations to Japanese and Swiss populations so far, we cannot entirely exclude that the haplotype $\mathrm{H} 1$ may also be present in unsampled populations of other parts of Asia where T. japonicus is present. Tracing the source of an introduction depends on the availability of information about population structure and may require analysis of more than one locus. To this end, a more comprehensive phylogeography study is underway which includes the $C O I$ barcode and microsatellite loci recently developed de novo in T. japonicus.

\section{Discussion}

Since the first detection of adventive T. japonicus in 2015, additional recoveries in the USA have shown that the adventive populations have established and are spreading. Given that T. japonicus occurs throughout the range of $H$. halys in its native range of eastern Asia, one potential outcome of the discovery of $T$. japonicus in Italy is that it will also establish and spread wherever $H$. halys has established in this region. Its recent discovery in the Ticino region of Switzerland (Stahl et al. 2018) lends support to this possibility.

Trissolcus mitsukurii is widespread in Asia, and its distribution extends to the southern limit of eastern Australia (Johnson 1991). Barcode sequences of Australian specimens of T. mitsukurii are not yet available and this region must be considered as a possible source of the Italian population. Of note, T. mitsukurii was introduced into Hawaii in 1966 as a biological control agent of Nezara viridula (L.) but apparently did not become established (Davis and Krauss 1967). This species has otherwise not been reported as an adventive parasitoid outside of its native range, but it is conceivable that its distribution will follow that of $H$. halys, as has occurred with T. japonicus. Continued surveys throughout the region will be needed to document their establishment and dispersal, in addition to determining their impact on $H$. halys populations and their interaction with native natural enemies in the region.

The phenomenon of parasitoids following in the footsteps of their invasive hosts has become a growing trend, particularly with species of Trissolcus Ashmead. In addition to discoveries of T. japonicus and T. mitsukurii in the invaded range of $H$. halys, an adventive population of T. hyalinipennis Rajmohana \& Narendran was found in California, USA, parasitizing eggs of the invasive bagrada bug, Bagrada hilaris (Burmeister) (Ganjisaffar et al. 2018). In each of these cases, the discovery was made through the cooperative effort of scientists working in the disciplines of biological control, taxonomy, and biological diversity, highlighting the synergy and necessity of collaboration.

Expansive ranges have recently been documented in platygastroid wasps that are not known to be of agricultural significance (Masner et al. 2009, Oliveira and Schoeninnger 2017, Popovici et al. 2018), but are charismatic and thus more easily recognized. As parasitoids are increasingly examined in the context of the world fauna, we expect to discover many more widespread species. 


\section{Acknowledgments}

We are grateful to Rosario Raso and Chiara Zampa for the assistance during field monitoring and egg mass sampling respectively in the sites of Codroipo and Cordenons and to Luca Tirinnanzi for assistance in the site of Lodi. We also thank Paride Dioli for the identification of the egg mass of the stink bug of the family Asopinae, Lucian Fusu (University of Iasi, Romania) for the identification of Anastatus bifasciatus, and Mircea Mitroiu (University of Iasi, Romania) for the ongoing analysis of the reared pteromalid specimens. This work was supported by the Florida Department of Agriculture and Consumer Services-Division of Plant Industry and the Italian Ministry of Agricultural Food and Forestry Policies (grant projects "Salvaolivi" DM 0033437 21/12/2017 and "Protezpiante" DM 0034140 29/12/2017). Taxonomic support was funded in part by USDA/APHIS Farm Bill award \#18-8130-0798-APHIS-IA to KAH/MCB/ EJT. USDA is an equal opportunity provider and employer.

\section{References}

Abram PK, Hoelmer KA, Acebes-Doria A, Andrews H, Beers EH, Bergh JC, Bessin R, Biddinger D, Botch P, Buffington ML, Cornelius ML, Costi E, Delfosse ES, Dieckhoff C, Dobson D, Donais D, Grieshop M, Hamilton G, Haye T, Hedstrom C, Herlihy MV, Hoddle MS, Hooks CRR, Jentsch P, Joshi NK, Kuhar TP, Lara J, Lee JC, Legrand A, Leskey TC, Lowenstein D, Maistrello L, Mathews CR, Milnes JM, Morrison III WR, Nielsen AN, Ogburn EC, Pickett CH, Poley K, Pote J, Radl J, Shrewsbury PM, Talamas EJ, Tavella L, Walgenbach JF, Waterworth R, Weber DC, Welty C, Wiman NG (2017) Indigenous arthropod natural enemies of the invasive brown marmorated stink bug in North America and Europe. Journal of Pest Science 90: 1009-1020. https://doi.org/10.1007/s10340-017-0891-7

Askew RR, Nieves-Aldrey JL (2014) Further observations on Eupelminae (Hymenoptera, Chalcidoidea, Eupelmidae) in the Iberian peninsula and Canary Islands, including descriptions of new species. Graellsia 60: 27-39.

Davis CJ, Krauss NLH (1967) Recent Introductions for Biological Control in Hawaii-XII. Proceedings of the Hawaiin Entomological Society 18: 375-380.

Dieckhoff C, Tatman K, Hoelmer KA (2017) Natural biological control of Halyomorpha halys (Hemiptera: Pentatomidae) by native egg parasitoids - a multi-year survey in northern Delaware. Journal of Pest Science 90: 1143-1158. https://doi.org/10.1007/s10340-017-0868-6

Faúndez EI, Rider DA (2017) The brown marmorated stink bug Halyomorpha halys (Stål, 1855) (Het.: Pentatomidae) in Chile. Arquivos Entomolóxicos 17: 305-307.

Folmer O, Black M, Hoeh W, Lutz R, Vrijenhoek R (1994) DNA primers for amplification of mitochondrial Cytochrome $\mathrm{C}$ oxidase subunit I from diverse metazoan invertebrates. Molecular Marine Biology and Biotechnology 3: 294-299.

Ganjisaffar F, Talamas EJ, Bon MC, Gonzalez L, Brown BV, Perring TM (2018) Trissolcus hyalinipennis Rajmohana \& Narendran (Hymenoptera, Scelionidae), a parasitoid of Bagrada hilaris (Burmeister) (Hemiptera, Pentatomidae), emerges in North America. Journal of Hymenoptera Research 65: 111-130. https://doi.org/10.3897/jhr.65.25620 
Gariepy TD, Haye T, Zhang J (2014) A molecular diagnostic tool for the preliminary assessment of host-parasitoid associations in biological control programmes for a new invasive pest. Molecular Ecology 23: 3912-3924. https://doi.org/10.1111/mec.12515

Haye T, Fischer S, Zhang J, Gariepy T (2015) Can native egg parasitoids adopt the invasive brown marmorated stink bug, Halyomorpha halys (Heteroptera: Pentatomidae), in Europe? Journal of Pest Science 88: 693-705. https://doi.org/10.1007/s10340-015-0671-1

Herlihy MV, Talamas EJ, Weber DC (2016) Attack and success of native and exotic parasitoids on eggs of Halyomorpha halys in three Maryland habitats. PLoS One 11(3): e0150275. https://doi.org/10.1371/journal.pone.0150275

Hoebeke ER, Carter ME (2003) Halyomorpha halys (Stål) (Heteroptera: Pentatomidae): A polyphagous plant pest from Asia newly detected in North America. Proceedings of the Entomological Society of Washington 105: 225-237.

Kalina V (1981) The Palearctic species of the genus Anastatus Motschulsky, 1860 (Hymenoptera, Chalcidoidea, Eupelmidae), with descriptions of new species. Silvaecultura Tropica et Subtropica1: 3-25.

Johnson NF (1991) Revision of Australasian Trissolcus species (Hymenoptera: Scelionidae). Invertebrate Taxonomy 5: 211-239. https://doi.org/10.1071/IT9910211

Kuhar T, Kamminga K (2017) Review of the chemical control research on Halyomorpha halys in the USA. Journal of Pest Science 90: 1021-1031. https://doi.org/10.1007/s10340017-0859-7

Lee DH, Short BD, Joseph SV, Bergh JC, Leskey TC (2013) Review of the biology, ecology, and management of Halyomorpha halys (Hemiptera: Pentatomidae) in China, Japan, and the Republic of Korea. Environmental Entomology 42: 627-641. https://doi.org/10.1603/ EN13006

Lee DH (2015) Current status of research progress on the biology and management of Halyomorpha halys (Hemiptera: Pentatomidae) as an invasive species. Applied Entomology and Zoology 50: 277-290. https://doi.org/10.1007/s13355-015-0350-y

Leigh JW, Bryant D (2015) POPART: Full-feature software for haplotype network construction. Methods in Ecology and Evolution 6: 1110-1116. https://doi.org/10.1111/2041210X.12410

Leskey TC, Nielsen AL (2018) Impact of the Invasive Brown Marmorated Stink Bug in North America and Europe: History, Biology, Ecology, and Management. Annual Review of Entomology 63: 599-618. https://doi.org/10.1146/annurev-ento-020117-043226

Masner L, Johnson NF, Musetti L (2009) Calliscelio elegans (Perkins), a tramp species, and a review of the status of the genus Caenoteleia Kieffer (Hymenoptera: Platygastridae). Zootaxa 2237: 59-66.

Matsuo K, Hirose Y, Johnson NF (2014) A taxonomic issue of two species of Trissolcus (Hymenoptera: Platygastridae) parasitic on eggs of the brown-winged green bug, Plautia stali (Hemiptera: Pentatomidae): resurrection of $T$. plautiae, a cryptic species of $T$. japonicus revealed by morphology, reproductive isolation and molecular evidence. Applied Entomology and Zoology 49: 385-394. https://doi.org/10.1007/s13355-014-0260-4

Mikó I, Vilhelmsen L, Johnson NF, Masner L, Pénzes Z (2007) Skeletomusculature of Scelionidae (Hymenoptera: Platygastroidea): head and mesosoma. Zootaxa 1571: 1-78. 
Milnes JM, Wiman NG, Talamas EJ, Brunner JF, Hoelmer KA, Buffington ML, Beers EH (2016) Discovery of an exotic eggparasitoid of the brown marmorated stink bug, Halyomorpha halys (Stål) in the Pacific Northwest. Proceedings of the Entomological Society of Washington 118: 466-470. https://doi.org/10.4289/0013-8797.118.3.466

Mita T, Nishimoto H, Shimizu N, Mizutani N (2014) Occurrence of Trissolcus basalis (Hymenoptera, Platygastridae), an egg parasitoid of Nezara viridula (Hemiptera, Pentatomidae), in Japan. Applied Entomology and Zoology 50: 27-31. https://doi.org/10.1007/s13355014-0298-3

Oliveira BG, Schoeninnger K (2017) First record of Mantibaria Kirby, 1900 (Hymenoptera, Scelionidae, Scelioninae) in the New World: a probable case of accidental introduction. Zootaxa 4237: 575-577. https://doi.org/10.11646/zootaxa.4237.3.8

Ormsby MD (2018) Technical Review - Proposed Treatments for BMSB (Halyomorpha halys (Stål); Pentatomidae). New Zealand Ministry for Primary Industries: Technical Document, 35pp.

Popovici OA, Masner L, Viciriuc M, Pintilioaie A, Notton DG, Talamas EJ (2018) New distribution data for some charismatic tramp species of Platygastroidea (Hymenoptera). Zootaxa 4370: 1-22. https://doi.org/10.11646/zootaxa.4370.1.1

Roversi PF, Binazzi F, Marianelli L, Costi E, Maistrello L, Sabbatini Peverieri G (2016) Searching for native egg-parasitoids of the invasive alien species Halyomorpha halys Stal (Heteroptera: Pentatomidae) in Southern Europe. Redia 99: 63-70.

Stahl J, Tortorici F, Pontini M, Bon MC, Hoelmer K, Marazzi C, Tavella L, Haye T (2018) First discovery of adventive populations of Trissolcus japonicus (Ashmead) in Europe. Journal of Pest Science. https://doi.org/10.1007/s10340-018-1061-2

Taekul C, Valerio AA, Austin AD, Klompen H, Johnson NF (2014) Molecular phylogeny of telenomine egg parasitoids (Hymenoptera: Platygastridae s.l.: Telenominae): evolution of host shifts and implications for classification. Systematic Entomology 39: 24-35. https:// doi.org/10.1111/syen.12032

Talamas EJ, Buffington ML, Hoelmer KA (2017) Revision of Palearctic Trissolcus Ashmead (Hymenoptera: Scelionidae). Journal of Hymenoptera Research 56: 3-185. https://doi. org/10.3897/jhr.56.10158

Talamas EJ, Herlihy MV, Dieckhoff C, Hoelmer KA, Buffington ML, Bon M-C, Weber DC (2015) Trissolcus japonicus (Ashmead) (Hymenoptera, Scelionidae) emerges in North America. Journal of Hymenoptera Research 43: 119-128. https://doi.org/10.3897/ JHR.43.4661

Wermelinger B, Wyniger D, Forster B (2008) First records of an invasive bug in Europe: $\mathrm{Ha}$ lyomorpha halys (Stål) (Heteroptera: Pentatomidae), a new pest on woody ornamentals and fruit trees? Mitteilungen der Schweizerischen Entomologischen Gesellschaft 81: 1-8.

Yang ZQ, Yao YX, Qiu LF, Li ZX (2009) A new species of Trissolcus (Hymenoptera: Scelionidae) parasitizing eggs of Halyomorpha halys (Heteroptera: Pentatomidae) in China with comments on its biology. Annals of the Entomological Society of America 102: 39-47. https://doi.org/10.1603/008.102.0104

Zhang J, Zhang F, Gariepy T, Mason P, Gillespie D, Talamas EJ, Haye T (2017) Seasonal parasitism and host specificity of Trissolcus japonicus in northern China. Journal of Pest Science 90: 1127-1142. https://doi.org/10.1007/s10340-017-0891-7 